\title{
Détermination du curium-242 par spectrométrie $\alpha$ et spectrométrie de masse dans les combustibles nucléaires irradiés
}

\author{
F. CHARTIER ${ }^{1}$, M. AUBERT ${ }^{1}$, J.-P. DEGROS ${ }^{1}$, J.-M. DUDA ${ }^{1}$
}

(Manuscrit reçu le 11 mars 2004, accepté le 12 juin 2004)

RÉSUMÉ Le curium-242 est un isotope particulièrement important dans les combustibles nucléaires irradiés en raison de sa contribution à la puissance résiduelle et également de sa chaîne de décroissance radioactive. La détermination de sa teneur est donc essentielle pour améliorer la connaissance des données nucléaires de base. Selon la nature et le temps de refroidissement du combustible irradié, la teneur en ${ }^{242} \mathrm{Cm}$ peut être inférieure à la limite de détection des techniques de spectrométrie de masse classiquement utilisées. La spectrométrie alpha peut alors prendre le relais pour ce radionucléide de courte période ( $T=162,9$ jours). À partir de solutions diluées de deux combustibles MOX (OXyde Mixte d'uranium et de plutonium) irradiés de faible temps de refroidissement et dont la teneur en ${ }^{242} \mathrm{Cm}$ est de quelques dizaines de pg, la mise au point de différentes séparations chimiques successives a permis d'obtenir des fractions de curium suffisamment pures pour effectuer l'analyse isotopique du curium par spectrométrie de masse à thermo-ionisation mais également la détermination du rapport ${ }^{242} \mathrm{Cm} /{ }^{244} \mathrm{Cm}$ par spectrométrie alpha. L'accord obtenu sur les rapports déterminés par les deux techniques a permis d'étendre la mesure par spectrométrie alpha à un combustible UOX (OXyde d'Uranium) de teneur en ${ }^{242} \mathrm{Cm}$ encore plus faible. L'association de la mesure du rapport ${ }^{242} \mathrm{Cm} /{ }^{244} \mathrm{Cm}$ avec le rapport ${ }^{244} \mathrm{Cm} /{ }^{238} \mathrm{U}$ déterminé par spectrométrie de masse à thermo-ionisation et dilution isotopique, à l'aide d'un traceur ${ }^{248} \mathrm{Cm} /{ }^{233} \mathrm{U}$, permet ainsi de connaître la teneur en curium-242 par rapport à l'uranium-238, utilisé comme référence, dans le combustible.

ABSTRACT Determination of curium-242 by $\alpha$ spectrometry and mass spectrometry in spent nuclear fuels.

Curium-242 is a particularly important isotope in spent nuclear fuels due to its contribution to the residual power and also of its radioactive decrease chain. The determination of its content is therefore essential to improve the knowledge of the nuclear data. According to the nature and the cooling time of the spent fuel, the ${ }^{242} \mathrm{Cm}$ content can be lower than the detection limit of the classically used mass spectrometric techniques. To overcome this limitation, alpha spectrometry can be advantageously used for this radionuclide of short period $(T=162.9$ days $)$. From dilute solutions of MOX fuels of short time of cooling and for which the content of ${ }^{242} \mathrm{Cm}$ is about a few tens of $\mathrm{pg}$, the development of different successive chemical separations permitted to get sufficiently pure curium fractions to perform curium isotopic analysis by thermal ionization mass spectrometry but also the determination of the ${ }^{242} \mathrm{Cm} /{ }^{244} \mathrm{Cm}$ ratio by alpha spectrometry. The agreement on the ratios determined by the two techniques has allowed to spread the measure by alpha

\footnotetext{
1 CEA Saclay, DEN/DPC/SECR/LANIE 91191 Gif/Yvette Cedex, France.
} 
spectrometry to a UOX fuel of content in ${ }^{242} \mathrm{Cm}$ even lower. The association of the ${ }^{242} \mathrm{Cm} /{ }^{244} \mathrm{Cm}$ ratio measurement with the ${ }^{244} \mathrm{Cm} /{ }^{238} \mathrm{U}$ ratio obtained by thermal ionization mass spectrometry and isotope dilution, with a ${ }^{248} \mathrm{Cm} /{ }^{233} \mathrm{U}$ spike, permits to know the content in curium-242 in relation to uranium-238 which is used as a reference in the fuel.

\section{Introduction}

Le curium-242 contribue significativement à la valeur de la puissance résiduelle totale des combustibles irradiés, en particulier dans les combustibles MOX: environ $22 \%$ de contribution à la valeur totale de la puissance résiduelle pour un temps de refroidissement de 1 an. Ce radionucléide est important également aux temps de refroidissement supérieurs de par sa décroissance alpha vers ${ }^{238} \mathrm{Pu}$ qui devient ainsi un des principaux contributeurs jusqu'à des temps de refroidissement de 300 ans.

D'autre part, le curium-242 intervient de façon privilégiée dans l'interprétation des calculs d'évolution du combustible (Allais, 1998 ; Roque, 2001) : il est le fils de l'américium-241 par capture de neutrons et le père du curium-243. La détermination de sa teneur absolue ou relative au ${ }^{238} \mathrm{U}$ permet donc d'avancer sur la connaissance des écarts calcul-expérience pour les radionucléides ${ }^{241} \mathrm{Am}$ et ${ }^{243} \mathrm{Cm}$ et d'améliorer ainsi la connaissance des données nucléaires de base.

Dans le domaine nucléaire, la mesure de la composition isotopique d'un élément est généralement effectuée par spectrométrie de masse à thermoionisation (TIMS) qui est l'une des meilleures techniques pour obtenir des rapports isotopiques reproductibles et justes (De Laeter, 1994 ; Heumann et al., 1995). Cependant, selon les radionucléides, le temps de refroidissement et la nature du combustible, cette technique peut ne pas être suffisamment sensible pour les mesurer. C'est, par exemple, le cas du ${ }^{242} \mathrm{Cm}$. La spectrométrie alpha peut alors prendre le relais pour cet émetteur alpha de courte période.

En raison, d'une part, des interférences isobariques en spectrométrie de masse et, d'autre part, des interférences en énergie en spectrométrie alpha, il est indispensable d'isoler au préalable le curium.

Après avoir décrit les étapes essentielles du développement de la séparation du curium (Chartier et al., 1999a), nous exposerons les méthodes utilisées en TIMS et en spectrométrie alpha pour déterminer le rapport ${ }^{242} \mathrm{Cm} /{ }^{244} \mathrm{Cm}$ dans deux combustibles MOX différents et de teneur en ${ }^{242} \mathrm{Cm}$ suffisamment élevée pour comparer les résultats obtenus par ces deux techniques. 
Nous verrons ensuite comment, pour un combustible UOX où seule la spectrométrie alpha permet la mesure du rapport ${ }^{242} \mathrm{Cm} /{ }^{244} \mathrm{Cm}$, l'association de ce résultat avec la détermination du rapport ${ }^{244} \mathrm{Cm} /{ }^{238} \mathrm{U}$ par double dilution isotopique en spectrométrie de masse permet de connaître la teneur en ${ }^{242} \mathrm{Cm}$ par rapport à l'uranium dans le combustible.

\section{Séparations élémentaires}

Les échantillons à caractériser sont obtenus après découpe des crayons de combustibles irradiés extraits des réacteurs et mise en solution par de l'acide nitrique concentré.

À partir des solutions initiales, l'uranium et le plutonium sont tout d'abord isolés, en boîte à gants, en raison de leurs concentrations importantes, à l'aide d'une résine échangeuse d'ions Dowex AG1X4. Les complexes nitrato-anioniques de $\mathrm{U}$ et de Pu sont fixés sur la résine tandis que les autres éléments ne sont pas retenus. On élue ensuite $\mathrm{U}$ et $\mathrm{Pu}$ avec des solutions aqueuses de concentrations différentes en $\mathrm{HNO}_{3}$. La fraction contenant l'uranium est ainsi prête à être caractérisée en isotopie par spectrométrie de masse, l'interférence isobarique à la masse $238(\mathrm{U}, \mathrm{Pu})$ ayant été supprimée.

La fraction non retenue par la résine est ensuite utilisée pour séparer par chromatographie liquide le curium de l'américium (interférences aux masses 242 et 243).

Le système chromatographique utilisé est constitué d'une pompe chromatographique (Gilson), d'une vanne six voies (Rhéodyne 7125) avec une boucle de $50 \mu \mathrm{L}$ et d'une colonne échangeuse de cations Hypersil SCX de $250 \times$ 4,6 mm (Interchim). La détection est effectuée à l'aide d'un détecteur UV-visible (Jasco) après dérivatisation post-colonne par de l'arsénazo III.

Seules les parties du système directement en contact avec les échantillons sont en boîte à gants.

La séparation mise au point est basée sur le principe d'un échange d'ions avec une phase stationnaire en silice greffée en $\mathrm{C} 18$ dont les greffons portent les sites échangeurs d'ions de type acide sulfonique. Une réaction de complexation auxiliaire, à l'aide de l'acide 2-hydroxy-2-méthylbutyrique (HMB), est couplée à la réaction d'échange d'ions et le pH est ajusté par une solution d'ammoniaque. 


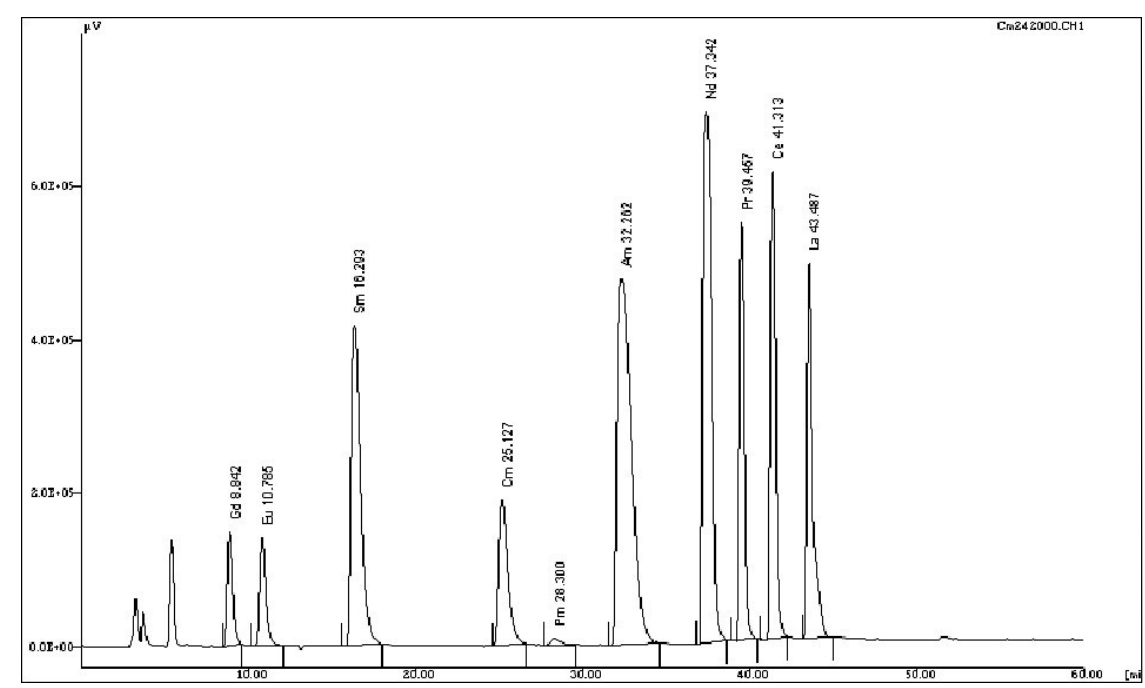

Figure 1 - Séparation chromatographique du curium (temps de rétention : $25 \mathrm{~min}$ ), $[\mathrm{HMB}]=0,1 \mathrm{~mol} \cdot \mathrm{L}^{-1}$, $p H=4,2 ;\left[\right.$ Arsénazo III] = 0,13 $\mathrm{mmol} \cdot \mathrm{L}^{-1}$. Détection UV-visible $(650 \mathrm{~nm})$.

Chromatographic separation of curium (retention time $: 25 \mathrm{~min}$ ), $[\mathrm{HMB}]=0.1 \mathrm{~mol} \cdot \mathrm{L}^{-1}$, $p H=4.2 ;$ Arsénazo III] $=0.13 \mathrm{mmol} \cdot \mathrm{L}^{-1} . U V$-visible detection $(650 \mathrm{~nm})$.

L'optimisation de la séparation a reposé sur l'étude de l'influence de la composition de la phase mobile et de son $\mathrm{pH}$ sur la rétention des éléments et sur la sélectivité, c'est-à-dire la différence de rétention, entre le curium et l'américium.

En imposant à la fois un temps de rétention raisonnable (inférieur à 30 minutes) et une sélectivité suffisamment importante, il est ressorti une seule condition d'analyse donnant des résultats satisfaisants : $[\mathrm{HMB}]=0,1 \mathrm{~mol} \cdot \mathrm{L}^{-1}, \mathrm{pH}=4,2$; permettant ainsi d'isoler le $\mathrm{Cm}$ qui est alors prêt à être analysé par TIMS. Le chromatogramme obtenu dans ces conditions expérimentales pour un échantillon de combustible MOX est montré figure 1 .

Pour la mesure des isotopes 242 et 244 du curium par spectrométrie alpha, à partir d'une dilution de la fraction recueillie (environ $200 \mathrm{kBq}$ de curium) une minéralisation est effectuée pour éliminer toute trace de HMB. Deux étapes supplémentaires de séparation sur résines échangeuses d'ions AG1X4 en milieu chlorhydrique, puis en milieu nitrique après passage du $\mathrm{Pu}$ au degré d'oxydation 4, sont effectuées afin d'obtenir une fraction de curium de haute pureté, exempte de traces d'interférants décelables. 


\section{TABLEAU I}

Rapports isotopiques du curium (en atomes) obtenus par TIMS dans les échantillons de combustibles irradiés. Moyenne de quatre mesures indépendantes. Incertitudes données à un niveau de confiance de $95 \%$.

Curium isotopic ratios (in atoms) determined by TIMS from spent nuclear fuels samples. Mean of four independent measurements, errors quoted with each ratio are at $95 \%$ confidence limits.

\begin{tabular}{cccc}
\hline Rapports isotopiques & MOX, échantillon 1 & MOX, échantillon 2 & UOX \\
\hline${ }^{242} \mathrm{Cm} /{ }^{244} \mathrm{Cm}$ & $0,001564 \pm 0,000003$ & $0,001015 \pm 0,000003$ & - \\
${ }^{243} \mathrm{Cm} /{ }^{244} \mathrm{Cm}$ & $0,01082 \pm 0,00003$ & $0,008546 \pm 0,000010$ & $0,006766 \pm 0,000020$ \\
${ }^{245} \mathrm{Cm} /{ }^{244} \mathrm{Cm}$ & $0,1393 \pm 0,0003$ & $0,1126 \pm 0,0002$ & $0,0879 \pm 0,0002$ \\
${ }^{246} \mathrm{Cm} /{ }^{244} \mathrm{Cm}$ & $0,01873 \pm 0,00004$ & $0,02522 \pm 0,00004$ & $0,02216 \pm 0,00009$ \\
\hline
\end{tabular}

\section{Analyse isotopique}

\subsection{Mesure de la composition isotopique du curium par TIMS dans des combustibles MOX et UOX}

Après séparation, la mesure des rapports isotopiques du curium est effectuée dans les deux échantillons de combustible MOX (isotopes 242 à 246) et dans l'échantillon de combustible UOX (isotopes 243 à 246), grâce à un spectromètre de masse à thermo-ionisation à secteur magnétique (Sector 54, GV Instrument) dont la source est isolée par une boîte à gants. Une masse de l'ordre de $40 \mathrm{ng}$ de curium contenant quelques dizaines de pg de ${ }^{242} \mathrm{Cm}$, est déposée sur le filament latéral en tantale d'un système à triple filaments. Le chauffage contrôlé des filaments dans la source permet la vaporisation puis l'atomisation et l'ionisation de l'élément. Un secteur magnétique sépare les ions en fonction de leur rapport masse sur charge et les isotopes du curium sont ensuite détectés de façon séquentielle à l'aide d'un détecteur de Daly, compte tenu de la faible intensité des courants d'ions à mesurer.

Les résultats de la mesure de l'isotopie du curium dans les trois échantillons sont présentés tableau I. Chaque résultat correspond à la moyenne de quatre mesures indépendantes. Les incertitudes associées, données à un niveau de confiance de $95 \%$, sont comprises entre 1,5 et 4,5 pour mille en relatif pour l'ensemble des rapports mesurés. Tous les résultats sont exprimés en rapport du nombre d'atomes de chacun des isotopes.

\subsection{Détermination du rapport ${ }^{242} \mathrm{Cm} /{ }^{244} \mathrm{Cm}$ par spectrométrie alpha dans les combustibles MOX et UOX}

Après les étapes de séparation chimique supplémentaires sur résines échangeuses d'ions AG1X4 en milieu chlorhydrique puis nitrique, nécessaires pour la mesure 


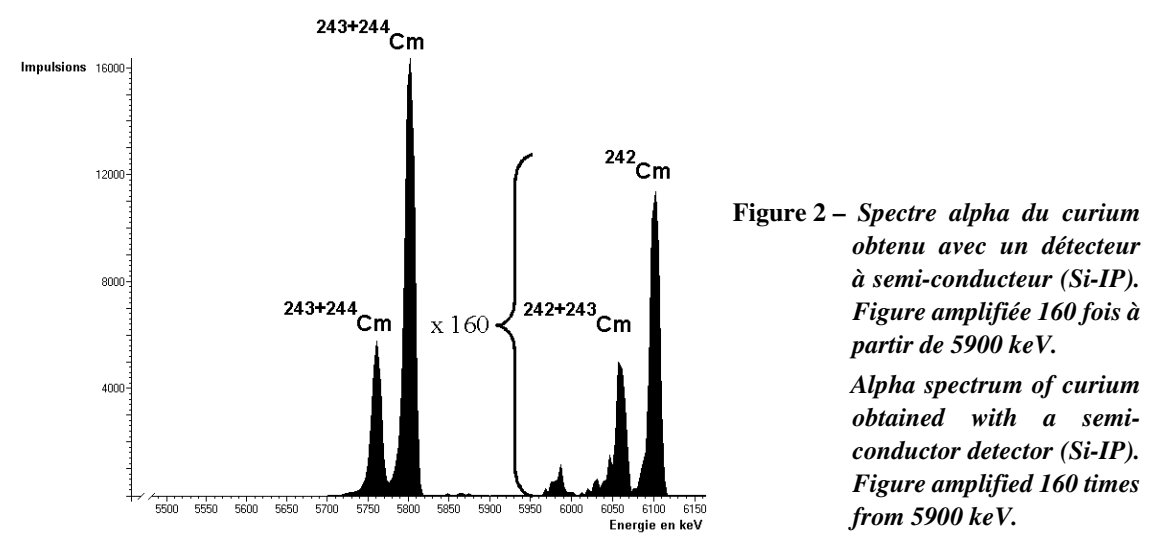

des isotopes ${ }^{242} \mathrm{Cm}$ et ${ }^{244} \mathrm{Cm}$ par spectrométrie alpha, le curium est fixé en couche mince homogène par électrodéposition à la surface d'un disque en acier inoxydable poli. Cette étape finale est importante, car elle permet d'éliminer toutes traces d'impuretés restantes qui auraient pour effet d'absorber une fraction de l'énergie des particules alpha émises par la source et par conséquent de dégrader la résolution en énergie du spectre.

Dans le cadre de cette étude, un semi-conducteur en silicium à jonction implantée passivée de $100 \mathrm{~mm}^{2}$ de surface, situé dans un module de spectrométrie alpha (INTERTECHNIQUE 7184) a été utilisé. Ce choix permet d'obtenir une résolution de $23 \mathrm{keV}$ à une énergie de $6 \mathrm{MeV}$ et par conséquent de limiter l'influence de l'interférence ${ }^{243} \mathrm{Cm}+{ }^{242} \mathrm{Cm}$ à $6,07 \mathrm{MeV}$ sur le pic ${ }^{242} \mathrm{Cm}$ à 6,11 MeV (Fig. 2).

Le rapport ${ }^{242} \mathrm{Cm} /{ }^{244} \mathrm{Cm}$ est déterminé à partir du rapport des activités spécifiques de ${ }^{242} \mathrm{Cm}$ et ${ }^{244} \mathrm{Cm}$. Pour un radionucléide donné, l'activité spécifique $A_{\mathrm{s}}$ est définie comme :

$$
A_{\mathrm{s}}=\frac{N}{M} \lambda=\frac{A_{\mathrm{ct}}}{m}=\frac{n_{\mathrm{E}}}{\varepsilon I_{\mathrm{E}} t_{\mathrm{c}} m}(\mathrm{~Bq} / \mathrm{g})
$$

avec $N$ : nombre d'Avogadro, $M$ : masse molaire, $\lambda$ : constante radioactive, $A_{\text {ct }}$ : activité déposée, $m:$ masse du radionucléide, $n_{\mathrm{E}}:$ nombre d'impulsions à l'énergie $E, \varepsilon$ : efficacité de détection, $I_{\mathrm{E}}$ : probabilité d'émission alpha à l'énergie $E$ et $t_{\mathrm{c}}$ : durée d'acquisition.

Finalement le rapport ${ }^{242} \mathrm{Cm} /{ }^{244} \mathrm{Cm}$ s'exprime simplement par le produit $\mathrm{du}$ rapport des périodes radioactives avec le rapport du nombre d'impulsions et de 


\section{TABLEAU II}

Rapports isotopiques ${ }^{242} \mathrm{Cm} /{ }^{244} \mathrm{Cm}$ (en atomes) obtenus par TIMS et par spectrométrie alpha pour deux échantillons différents de combustibles MOX et par spectrométrie alpha pour un échantillon de combustible UOX. Incertitudes données à un niveau de confiance de $95 \%$. Les résultats sont ramenés à la même date pour comparaison.

${ }^{242} \mathrm{Cm} /{ }^{244} \mathrm{Cm}$ isotope ratio (in atoms) obtained by TIMS and by alpha spectrometry for two different samples of MOX fuel and by alpha spectrometry for a sample of UOX fuel. Errors quoted with each ratio are at $95 \%$ confidence limits. Results are given at the same date for comparison.

\begin{tabular}{cccc}
\hline${ }^{242} \mathrm{Cm} /{ }^{244} \mathrm{Cm}$ & MOX, échantillon 1 & MOX, échantillon 2 & UOX \\
\hline TIMS & $0,001564 \pm 0,000003$ & $0,001015 \pm 0,000003$ & - \\
Spectrométrie alpha & $0,001567 \pm 0,000061$ & $0,001029 \pm 0,000039$ & $0,000095 \pm 0,000004$ \\
Écart relatif $(\%)$ & 0,19 & 1,1 & - \\
\hline
\end{tabular}

l'inverse du rapport des probabilités d'émission. Il est indépendant de l'efficacité de détection et de la durée d'acquisition :

$$
\frac{{ }^{242} \mathrm{Cm}}{{ }^{244} \mathrm{Cm}}=\frac{M_{4} m_{2}}{M_{2} m_{4}}=\frac{T_{2} I_{4} n_{2}}{T_{4} I_{2} n_{4}}
$$

où $T_{2}$ et $T_{4}$ sont les périodes radioactives de ${ }^{242} \mathrm{Cm}$ et ${ }^{244} \mathrm{Cm}$.

L'incertitude sur la mesure du rapport ${ }^{242} \mathrm{Cm} /{ }^{244} \mathrm{Cm}$ est pilotée par le nombre d'impulsions détectées dans les raies d'absorption, en particulier par le nombre d'impulsions dues au ${ }^{242} \mathrm{Cm}$. Par exemple, dans le cas du combustible UOX, l'activité de ce radionucléide est 200 fois plus faible que l'activité $\mathrm{du}{ }^{244} \mathrm{Cm}$. Il en résulte que l'incertitude relative sur le nombre d'impulsions dues au ${ }^{242} \mathrm{Cm}$ est multipliée d'un facteur 15 par rapport à l'incertitude relative de ${ }^{244} \mathrm{Cm}$. Seul l'accroissement de la durée d'acquisition permet de minimiser l'incertitude sur le ${ }^{242} \mathrm{Cm}$.

\subsection{Comparaison des résultats obtenus par les deux techniques}

Les résultats de mesure des rapport ${ }^{242} \mathrm{Cm} /{ }^{244} \mathrm{Cm}$ obtenus par TIMS et par spectrométrie alpha pour les deux échantillons de combustible MOX sont reportés tableau II. Les écarts relatifs obtenus entre les deux techniques, pour les deux échantillons, sont respectivement de $0,2 \%$ et $1,1 \%$. L'incertitude est inférieure à $0,3 \%$ (niveau de confiance de $95 \%$ ) en TIMS et de 3,9\% en spectrométrie alpha (niveau de confiance de $95 \%$, facteur d'élargissement $k=2$ ), cette dernière incertitude étant directement liée à la durée d'acquisition.

Cet accord montre l'absence d'interférences pour chacune des techniques et permet de retenir l'utilisation de la spectrométrie alpha pour déterminer le rapport ${ }^{242} \mathrm{Cm} /{ }^{244} \mathrm{Cm}$ dans un combustible UOX. 


\section{TABLEAU III}

Concentrations et abondances isotopiques des isotopes traceurs (moyenne de 8 mesures indépendantes, niveau de confiance de $95 \%$ ).

Concentrations and isotopes abundances of spike isotopes (mean of 8 independent measurements, $95 \%$ confidence limits).

\begin{tabular}{ccc}
\hline & \multicolumn{2}{c}{ Traceur $\mathrm{Cm}-\mathrm{U}$} \\
\hline Isotope enrichi & ${ }^{248} \mathrm{Cm}$ & ${ }^{233} \mathrm{U}$ \\
Abondance de l'isotope traceur $(\%$ at. $)$ & $89.511 \pm 0.010$ & $99.537 \pm 0.005$ \\
Concentration de l'isotope traceur $(\mu \mathrm{g} / \mathrm{g})$ & $0.7347 \pm 0.0012$ & $1034.8 \pm 1.5$ \\
Rapport isotopique (at.) & ${ }^{244} \mathrm{Cm} /{ }^{248} \mathrm{Cm}$ & ${ }^{238} \mathrm{U} /{ }^{233} \mathrm{U}$ \\
& $0.001353 \pm 0.000007$ & $0.000208 \pm 0.000002$ \\
\hline
\end{tabular}

Les résultats obtenus par spectrométrie alpha pour ce combustible (Tab. II) correspondent à environ $1,7 \mathrm{~Bq}$ soit $0,014 \mathrm{pg}$ de ${ }^{242} \mathrm{Cm}$. Compte tenu de la faible quantité de ${ }^{242} \mathrm{Cm}$ déposée, la durée d'acquisition a été augmentée de façon à obtenir une incertitude de l'ordre de $4 \%(k=2)$.

\section{Détermination des rapports $\mathrm{Cm} /{ }^{238} \mathrm{U}$ par dilution isotopique}

Le principe de la méthode (Heumann, 1992 ; Chartier et al., 1999a, 1999b) est d'ajouter à l'élément à doser, une quantité connue du même élément de composition isotopique différente, le traceur. Connaissant l'isotopie et la concentration du traceur, la mesure des abondances isotopiques des éléments dans l'échantillon et dans les mélanges échantillon-traceur permet, par l'intermédiaire des équations de bilan isotopique, d'obtenir la concentration initiale de l'élément avec une justesse qui dépend de la justesse de la mesure des rapports isotopiques, soit quelques pour mille par TIMS.

Un autre avantage de cette technique est que le rendement de séparation chimique n'a pas besoin d'être constant, ni même connu précisément, puisque seuls des rapports isotopiques sont mesurés.

La méthode de dilution isotopique à l'aide d'un traceur double permet, quant à elle, de mesurer de façon très précise le rapport de concentration de deux éléments et n'est également basée que sur la mesure de rapports isotopiques.

Cette méthode est employée afin d'obtenir directement les rapports de concentration ${ }^{244} \mathrm{Cm} /{ }^{238} \mathrm{U}$ avec la meilleure précision possible. Un traceur double ${ }^{248} \mathrm{Cm} /{ }^{233} \mathrm{U}$, caractérisé en isotopie et concentration (Tab. III), a été utilisé dans ce but. Ce traceur a été préparé puis caractérisé en isotopie et en concentration par dilution isotopique inverse en TIMS sur des séries de mélanges traceur-référence. Les solutions de référence étant dans ce cas une solution de ${ }^{244} \mathrm{Cm}$ certifiée en 
activité et une solution ${ }^{235} \mathrm{U}$ (NIST SRM U930) préparée par pesée. Huit mélanges ${ }^{248} \mathrm{Cm}_{-}{ }^{244} \mathrm{Cm}$ et ${ }^{233} \mathrm{U}_{-}{ }^{235} \mathrm{U}$ ont ainsi été analysés par TIMS après séparation chimique de $\mathrm{Cm}$ et de $\mathrm{U}$.

Avec cette solution de traceur double, le rapport ${ }^{244} \mathrm{Cm} /{ }^{238} \mathrm{U}$ dans les échantillons de combustible irradié peut être déterminé en utilisant l'équation de bilan isotopique :

$$
\left(\frac{{ }^{244} \mathrm{Cm}}{{ }^{238} \mathrm{U}}\right)_{\mathrm{S}}=\left(\frac{{ }^{248} \mathrm{Cm}}{{ }^{233} \mathrm{U}}\right)_{\mathrm{Sp}} \times \frac{\left[\left(\frac{{ }^{244} \mathrm{Cm}}{{ }^{248} \mathrm{Cm}}\right)_{\mathrm{M}}-\left(\frac{{ }^{244} \mathrm{Cm}}{{ }^{248} \mathrm{Cm}}\right)_{\mathrm{Sp}}\right] \times\left[1-\left(\frac{2{ }^{238} \mathrm{U}}{{ }^{233} \mathrm{U}}\right)_{\mathrm{M}} \times\left(\frac{{ }^{233} \mathrm{U}}{{ }^{238} \mathrm{U}}\right)_{\mathrm{S}}\right]}{\left[\left(\frac{{ }^{233} \mathrm{U}}{{ }^{233}}\right)_{\mathrm{M}}-\left(\frac{{ }^{238} \mathrm{U}}{{ }^{233} \mathrm{U}}\right)_{\mathrm{Sp}}\right] \times\left[1-\left(\frac{{ }^{244} \mathrm{Cm}}{{ }^{248} \mathrm{Cm}}\right)_{\mathrm{M}} \times\left(\frac{{ }^{248} \mathrm{Cm}}{{ }^{244} \mathrm{Cm}}\right)_{\mathrm{S}}\right]}
$$

avec $\mathrm{S}$ : échantillon, $\mathrm{M}$ : mélange échantillon-traceur et $\mathrm{Sp}$ : traceur (tous les rapports sont exprimés en atomes).

Compte tenu du choix des isotopes dans la fabrication du traceur $\left({ }^{248} \mathrm{Cm}\right.$ et ${ }^{233} \mathrm{U}$ sont absents des échantillons de combustibles), l'équation se simplifie. Seule la mesure des rapports ${ }^{244} \mathrm{Cm} /{ }^{248} \mathrm{Cm}$ et ${ }^{233} \mathrm{U} /{ }^{238} \mathrm{U}$ dans les mélanges échantillontraceur est directement impliquée dans la détermination finale du rapport ${ }^{244} \mathrm{Cm} /{ }^{238} \mathrm{U}$.

Après séparations par chromatographie, les fractions purifiées de $\mathrm{U}$ et $\mathrm{Cm}$ dans les différents mélanges sont analysées par TIMS en multi-collection statique par cages de Faraday, avec des dépôts respectifs de $2 \mu \mathrm{g}$ et $40 \mathrm{ng}$. Dans chaque cas, les quantités d'échantillon et de traceur sont choisies de façon à obtenir des rapports isotopiques proches de 1 dans les mélanges, ce qui permet d'atteindre des reproductibilités de l'ordre de $0,01 \%$.

Avec ces résultats et les caractéristiques du traceur, on obtient directement le rapport ${ }^{244} \mathrm{Cm} /{ }^{238} \mathrm{U}$ dans les échantillons (Tab. IV) avec une incertitude, calculée par la théorie de la propagation des erreurs (De Bièvre et Debus, 1965), de l'ordre de 3 pour mille (niveau de confiance de $95 \%$ ). Les rapports ${ }^{242} \mathrm{Cm} /{ }^{238} \mathrm{U}$ sont ensuite calculés à l'aide des rapports ${ }^{242} \mathrm{Cm} /{ }^{244} \mathrm{Cm}$ déterminés par TIMS pour les deux échantillons MOX et par spectrométrie alpha pour les trois échantillons. Les incertitudes sur les rapports ${ }^{242} \mathrm{Cm} /{ }^{238} \mathrm{U}$ obtenus par TIMS sont alors de 3 à 5 pour mille et de l'ordre de $4 \%$ sur les mêmes rapports déterminés par spectrométrie alpha, toujours au niveau de confiance de $95 \%$. 


\section{TABLEAU IV}

Rapports du nombre d'atomes de ${ }^{244} \mathrm{Cm}$ et de ${ }^{242} \mathrm{Cm}$ formés par rapport au nombre d'atomes $\mathrm{de}^{238} \mathrm{U}$ dans les échantillons. Mesures effectuées par TIMS (moyenne de 4 mesures indépendantes, niveau de confiance de $95 \%$ ) et/ou par spectrométrie alpha (niveau de confiance de $95 \%$ ).

${ }^{242} \mathrm{Cm}$ and ${ }^{244} \mathrm{Cm}$ atom numbers formed with respect to ${ }^{238} \mathrm{U}$ atom number in the samples. Measurements performed by TIMS (mean of 4 independent measurements, $95 \%$ confidence limits) and/or by alpha spectrometry (95\% confidence limits).

\begin{tabular}{cccc}
\hline Rapports isotopiques & MOX, échantillon 1 & MOX, échantillon 2 & UOX \\
\hline${ }^{244} \mathrm{Cm} /{ }^{238} \mathrm{U}$ (TIMS) & $1086 \times 10^{-6} \pm 3 \times 10^{-6}$ & $1088 \times 10^{-6} \pm 3 \times 10^{-6}$ & $270,9 \times 10^{-6} \pm 0,8 \times 10^{-6}$ \\
${ }^{242} \mathrm{Cm} /{ }^{238} \mathrm{U}$ (TIMS) & $1,699 \times 10^{-6} \pm 0,005 \times 10^{-6}$ & $1,104 \times 10^{-6} \pm 0,004 \times 10^{-6}$ & - \\
${ }^{242} \mathrm{Cm} /{ }^{238} \mathrm{U}$ (Sp. alpha) & $1,702 \times 10^{-6} \pm 0,066 \times 10^{-6}$ & $1,116 \times 10^{-6} \pm 0,042 \times 10^{-6}$ & $0,0344 \times 10^{-6} \pm 0,0011 \times 10^{-6}$
\end{tabular}

\section{Conclusion}

Dans le but de déterminer le rapport ${ }^{242} \mathrm{Cm} /{ }^{238} \mathrm{U}$ dans des solutions de combustibles irradiés, une succession de séparations chimiques par résines échangeuses d'ions et par chromatographie d'échange d'ions des différents actinides a été développée. Ces séparations permettent d'obtenir des fractions de curium purifiées et d'éliminer ainsi les interférences isobariques en spectrométrie de masse et les interférences en énergie en spectrométrie alpha.

La détermination du rapport ${ }^{242} \mathrm{Cm} /{ }^{244} \mathrm{Cm}$ par spectrométrie de masse à thermo-ionisation et par spectrométrie alpha dans deux échantillons différents de combustible MOX a montré un écart entre les deux techniques de respectivement $0,2 \%$ et $1,1 \%$ avec une incertitude de l'ordre $3,9 \%$ au niveau de confiance de $95 \%$, ce qui a permis de retenir l'emploi de la spectrométrie alpha pour la mesure du rapport ${ }^{242} \mathrm{Cm} /{ }^{244} \mathrm{Cm}$ dans un combustible UOX de teneur en ${ }^{242} \mathrm{Cm}$ trop faible pour être mesurée par TIMS.

L'association de cette détermination par spectrométrie alpha avec la méthode de la double dilution isotopique en TIMS a permis de déterminer le rapport ${ }^{242} \mathrm{Cm} /{ }^{238} \mathrm{U}$ dans une solution de combustible UOX avec une incertitude finale correspondant à celle de la mesure du rapport ${ }^{242} \mathrm{Cm} /{ }^{244} \mathrm{Cm}$ par spectrométrie alpha, soit environ $4 \%$.

L'association des techniques de mesures nucléaires et de la spectrométrie de masse permet ainsi la mesure des rapports isotopiques d'un radioélément comme le curium dont les isotopes ont des périodes très différentes. 


\section{RÉFÉRENCES}

Allais V. (1998) Qualification du formulaire DARWIN pour les études du cycle du combustible pour les réacteurs à eau bouillante. Thèse de doctorat, Université de Provence.

Chartier F., Aubert M., Pilier M. (1999a) Determination of Am and Cm in spent nuclear fuels by isotope dilution inductively coupled plasma mass spectrometry and isotope dilution thermal ionization mass spectrometry after separation by high-performance liquid chromatography, Fresenius'J. Anal. Chem. 364, 320-327.

Chartier F., Salmon M., Tabarant M., Tran B. (1999b) Determination of erbium in nuclear fuels by isotope dilution thermal ionization mass spectrometry and glow discharge mass spectrometry, J. Anal. Atom. Spectrom. 14, 1461-1465.

De Bièvre P.J., Debus G.H. (1965) Precision mass spectrometric isotope dilution analysis, Nucl. Instrum. and Meth. 32, 224-228.

De Laeter J.R. (1994) Role of isotope mass spectrometry in cosmic abundance studies, Mass Spectrom. Rev. 13, 3-22.

Heumann K.G. (1992) Isotope dilution mass spectrometry (IDMS) of the elements, Mass Spectrom. Rev. 11, 41-67.

Heumann K.G., Eisenhut S., Gallus S., Hebeda E.H., Nusko R., Vengosh A., Walczyk T. (1995) Recent developments in thermal ionization mass spectrometric techniques for isotope analysis, Analyst 120, 1291-1299.

Roque B. (2001) Rapport de qualification du formulaire du cycle du combustible DARWIN 1.4 pour le calcul du bilan matière des combustibles REP UOX et MOX. CEA centre de Cadarache, Rapport Technique SPRC/LECy/01.139. 